\title{
Analysis of M/G/1 Feedback Queue under Steady State When Catastrophes Occur
}

\author{
S.Shanmugasundaram ${ }^{1}$, G.Sivaram ${ }^{2}$, \\ ${ }^{1}$ Assistant Professor, Department of Mathematics, Government Arts College, Salem- 636007 , India \\ Email: ssundaramsss@rediffmail.com \\ ${ }^{2}$ Associate Professor, Department of Mathematics,Government Arts College, Salem- 636 007, India \\ Email: gsivaram1965@gmail.com
}

Article History: Received: 11 January 2021; Accepted: 27 February 2021; Published online: 5 April 2021

Abstract: In this paper we analyse the M/G/1 feedback queue under steady states conditions
when catastrophe occur. The stationary probability of ' $\mathrm{n}$ ' and zero customers sins the system are derived. The
asymptotic behaviour of the model and the averages queues length
are also obtained. The numerical example are provided to test the feasibility of the model.

Keywords: Bernoulli process, customer, feedback, catastrophes, stationary distribution, asymptotic behaviour

\section{INTRODUCTION}

A queue is a waiting line which demands service from a server.The queue does not include a customer being serviced. Queueing mathematician A.K. Erlang. The Erlang work[1] on queueing stimulated many authors to develop a variety of queueing models. Many queueing situations have features that the customer may be serviced once again. If a customer is not satisfied by his service or he expects more service then he joins the queue to get additional service is called feedback. The customer may (or) may not opt for a feedback. In the year 1963 Takacs [8] first introduced the concept of feedback mechanism in queues. In 1996 Gautam Choudhary and Madhu Chandapaul [3] have proposed a two phase queueing system with Bernoulli feedback.

In certain queueing models before starting a service, the server may have to do some preparatory work or some alignment must be done in the case of certain necessities. This sort of preparatory work for customers occur in hospitals, production process, bank etc. Santhakumaran and Thangaraj [7] have proposed a single server queue with impatient and feedback customers. Santhakumaran and Shanmugasundaram [6 ] have preparatory work on arriving customers with a single server feedback queue. Santhakumaran, Ramasamy and Shanmuga sundaram[13] have also studied a single queue with instantaneous Bernoulli feedback and setup time. Thangaraj and Vanitha [12] have focussed on a continued fraction approach to a $\mathrm{M} / \mathrm{M} / 1$ queue with feedback.Chandrasekaran and Saravanarajan[14] made a study on transient and reliability analysis of $\mathrm{M} / \mathrm{M} / 1$ feedback queue subject to catastrophes, server failures and repairs.In queueing system catastrophes means sudden calamity that occur in queue or service facility. When catastrophes occur in the system, allthe available customers are destroyed immediately and the server gets inactivated. Catastrophes modelling and analysis has been playing a vital role in various areas of science and technology. Chao [2] has modelled a queueing network mode 1 with catastrophes and product form solution. Shanmugasundaram and Chitra [9] have made a study on time dependent solution of a single server feedback queue customer has a service with and without preparatory work when catastrophes occur. Krishnakumar, Krishnamoorthy, Pavai Madheswari and Sadiq Basha [15] studied a transient analysis of a single server queue with catastrophes, failures and repairs.

Krishnakumar, Arivudainambi [10] focussed on transient state solution to a M/M/1 queue with catastrophes. Parthasarathy [11 ] made a study on a transient solution to a M/M/1 queue. Jain and kumar [4 ] have studied on M/G/1 queue with catastrophes. Krishnakumar and Pavai Madheswari [5] have made a study on transient analysis of an $\mathrm{M} / \mathrm{M} / 1$ queue subject to catastrophes and server failures.

\section{Description of the system}


In this model,external customers arrive accordingto a poisson process with rate $\lambda$. The service follows general discipline with service rate $\mu$ and the service for an arriving customer begins instantaneously if the server is idle upon an arrival.

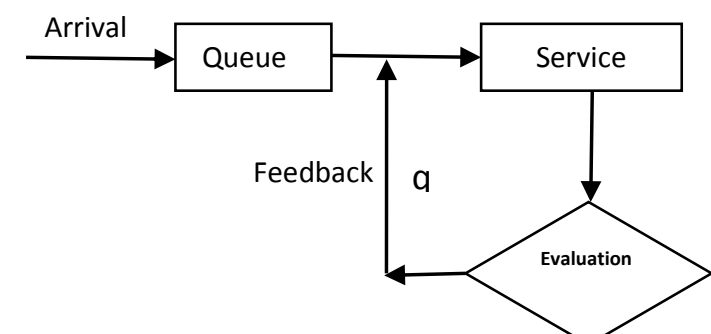

After getting service, the customer makes a decision depending on the level of service whether to depart (or) Departurefeqalback. If the customer does feedback, he joins $\downarrow$ the feedback stream with probability $q$ and joins the end of the queue. If a customer does not feedback,he joins the departure process with probability $p$ so that $p+q=1$. The queue discipline is FIFO and the capacity of the queue is infinite. Catastrophes occur from arrival the and the service process with rate $\Omega$. When catastrophes occur, all the available customers are destroyed immediately and the server becomes inactive. The server will be ready for service at the time of a new arrival. The motivation for this model comes from bank, hospital, production systems, restaurant etc. Let $P_{n}(t)=P[x(t)=n], n=0,1,2, \ldots$. denote the probabilities that there are 'n'customers in the system at time $\mathrm{t}$ and let $\mathrm{P}(\mathrm{x}, \mathrm{t})=\sum_{0}^{\infty} P(t) x^{n}$ be its probability generating function. Assume that there are no customers in the system at time $t=0$. i.e., $\quad P_{0}(0)=1$ The system of differential difference equations for the probability $\mathrm{P}_{\mathrm{n}}$ is

$$
-\lambda \mathrm{P}_{0}+\mu \mathrm{P}_{1}+\Omega\left(1-\mathrm{P}_{0}\right)=0
$$

and for $\mathrm{n}=1,2,3, \ldots \ldots \ldots$

$$
-\lambda P_{n-1}-(\lambda+\mu+\Omega) P_{n}+\mu P_{n+1}=0
$$

\section{Theorem :1}

$$
\begin{aligned}
& \text { The } \quad \begin{array}{l}
\text { Stationary } \\
\text { \{ } \pi_{n} \\
\text { catastrophes occur is } \quad n \geq 0 \text { for }
\end{array} \\
& \pi_{0}=1-\rho \\
& \pi_{n}=(1-\rho) \rho^{\mathrm{n}}, \quad \mathrm{n}=1,2, \ldots \ldots \ldots \ldots
\end{aligned}
$$$$
\text { Where } \rho=\left[\frac{(\lambda+\mu+\Omega)-\sqrt{\lambda^{2}+\mu^{2}+\Omega^{2}+2(\lambda \Omega+\mu \Omega-\lambda \mu)}}{2 \mu}\right]
$$

Proof :The Laplace transform of the steady system is

$$
P_{0}^{\prime}(x)=\frac{1+\frac{\Omega}{x}}{(x+\lambda+\Omega)-\left(\frac{w-\sqrt{w^{2}-4 \lambda \mu}}{2}\right)}
$$

Where $w=(x+\lambda+\mu+\Omega)$

$$
\pi_{0} \quad=\lim _{x \rightarrow 0} x P_{0}^{*}(x)
$$




$$
\begin{gathered}
=\lim _{x \rightarrow 0} \frac{x+\Omega}{(x+\lambda+\Omega)-\left(\frac{w-\sqrt{w^{2}-4 \lambda \mu}}{2}\right)} \\
=\frac{2 \Omega}{2(\lambda+\Omega)-\left[(\lambda+\mu+\Omega)+\sqrt{(\lambda+\mu+\Omega)^{2}-4 \lambda \mu}\right]} \\
=\frac{2 \Omega}{(\lambda-\mu+\Omega)+\sqrt{(\lambda+\mu+\Omega)^{2}-4 \lambda \mu}} \\
\pi_{0}=1-\left[\frac{(\lambda+\mu+\Omega)-\sqrt{\lambda^{2}+\mu^{2}+\Omega^{2}+2 \lambda \Omega+2 \mu \Omega-2 \lambda \mu}}{2 \mu}\right]
\end{gathered}
$$

Also taking Laplace transform of the steady state probability for $n$ customers in the system, we obtain

$$
\begin{gathered}
\lim _{x \rightarrow 0} x P_{n}^{\prime}(x)=\left(\frac{w-\sqrt{w^{2}-4 \lambda \mu}}{2 \mu}\right)^{n} \lim _{x \rightarrow 0} x P_{0}^{*}(x) \\
\pi_{n}=\lim _{x \rightarrow 0}\left(\frac{w-\sqrt{w^{2}-4 \lambda \mu}}{2 \mu}\right)^{n} \pi_{0} \\
\pi_{n}=\pi_{0}\left[\frac{(\lambda+\mu+\Omega)-\sqrt{\lambda^{2}+\mu^{2}+\Omega^{2}+2 \lambda \Omega+2 \mu \Omega-2 \lambda \mu}}{2 \mu}\right]^{n}
\end{gathered}
$$

$\pi_{n}=(1-\rho) \rho^{n}, \quad n=1,2,3 \ldots \quad$ and $\quad$ the stationary probability distribution exists if and only if $\rho<1$.

Theorem 2

The asymptotic behaviour of average queue length $H(t)$ when $\Omega>0$ is $H(t)=\left(\frac{\lambda-\mu}{\Omega}\right)+\frac{2 \mu}{2(\lambda+\Omega)-\left[(\lambda-\mu+\Omega)-\sqrt{(\lambda+\mu+\Omega)^{2}-4 \lambda \mu}\right]}$ as $\quad t \rightarrow \infty$.

Proof: Consider the function $P(x, t)=\sum_{n=0}^{\infty} P_{n}(t) x^{n}$ together probability generating initial conditions and using the equations (1) and (2), the probability generating function $P(x, t)$ becomes

$$
\frac{\partial P(x, t)}{\partial t}=\left[\lambda+\frac{\mu}{x}-(\lambda+\mu+\Omega)\right] P(x, t)+\mu\left(1-\frac{1}{x}\right) P_{0}+\Omega
$$

The average queue length is

$$
\begin{aligned}
H(t)=\sum_{n=1}^{\infty} n P_{n}(t)=\frac{\partial P(x, t)}{\partial t} \quad \text { at } \quad x=1 \\
\\
\quad \frac{d H(t)}{d t}+\Omega h(t)=\lambda-\mu\left(1-P_{0}\right)
\end{aligned}
$$

This differential equation is linear in $H(t)$ and solving for $H(t)$ we get

$$
\begin{gathered}
H(t) e^{\int \Omega d t}=\int_{0}^{t}\left[\lambda-\mu\left(1-P_{0}\right)\right] e^{\int \Omega d t} d t+c \\
H(t)=\frac{\lambda}{\Omega}\left(1-e^{\Omega t}\right)-\frac{\mu}{\Omega}\left(1-e^{\Omega t}\right)+\mu \int_{0}^{t} P_{0}(u) e^{-\Omega(t-u)} d u
\end{gathered}
$$

Taking Laplace transform for the above expression, we get 


$$
\begin{gathered}
H^{*}(x)=\frac{\lambda}{\mathrm{x}(\mathrm{x}+\Omega)}-\frac{\mu}{x(x+\Omega)}+\frac{P}{(x+\Omega)} P_{0}^{*}(x) \\
\lim _{t \rightarrow \infty} H(t)=\lim _{x \rightarrow 0} x H^{*}(x)=\lim _{x \rightarrow 0} \frac{\lambda-\mu(t)}{x+\Omega} \\
+\frac{\mu(t)}{(x+\lambda+\Omega)-\left[\frac{(x+\lambda+\mu(t)+\Omega)-\sqrt{(x+\lambda+\mu(t)+\Omega)^{2}-4 \lambda \mu(t)}}{2}\right]} \\
=\frac{\lambda-\mu(t)}{\Omega}+\frac{2 \mu(\mathrm{t})}{2(\lambda+\Omega)-\left[(\lambda+\mu(t)+\Omega)-\sqrt{(\lambda+\mu(t)+\Omega)^{2}-4 \lambda \mu(t)}\right]}
\end{gathered}
$$

as $\quad t \rightarrow \infty$

$$
H(t)=\left(\frac{\lambda-\mu}{\Omega}\right)+\frac{2 \mu}{2(\lambda+\Omega)-\left[(\lambda-\mu+\Omega)-\sqrt{(\lambda+\mu+\Omega)^{2}-4 \lambda \mu}\right]}
$$

If the service is carried out with and without preparatory work and therespective service rates are taken as $\mu_{1}, \mu_{2}$ and if $\mathrm{p}$ is the probability for preparatory work and $\mathrm{q} \quad$ is $\mu_{1}$ the probability without preparatory work such that $\mathrm{p}+\mathrm{q}=1$, then the asymptotic behaviour of average queue length $H=\left(\frac{\lambda-\left(p \mu_{1}+q \mu_{2}\right)}{\Omega}\right)$

$$
+\frac{2\left(p \mu_{1}+q \mu_{2}\right)}{2(\lambda+\Omega)-\left[\left(\lambda-\left(p \mu_{1}+q \mu_{2}\right)+\Omega\right)-\sqrt{\left(\lambda+\left(p \mu_{1}+q \mu_{2}\right)+\Omega\right)^{2}-4 \lambda\left(p \mu_{1}+q \mu_{2}\right)}\right]}
$$

If the customers without preparatory work areonly allowed to feedback with probability $q$ but the customers with preparatory work are not allowed to feedback and depart from the system with probability $\mathrm{p}$ such that $\mathrm{p}+\mathrm{q}=1$ with service rates $\mu_{1}$ and $\mu_{2}$ respectively then also the symptotic behaviour of the average queue length coincides with the result of Shanmugasundaram and Chitra.

Numerical Study

In this section a numerical study is made based on the average queue length of the model. For this purpose two tables are computed by varying the values of $\lambda$ and $\Omega$ by keeping $\mu$ fixed and then varying $\mu$ and $\Omega$ by keeping $\lambda$ fixed with $\lambda<\mu$.

Table :1

The average length of the system $\mathrm{H}(\mathrm{t})$ is computed for catastrophic effect of $\Omega=0.3,0.6,0.9$ with $\mu=10$.

\begin{tabular}{|c|c|c|c|}
\hline$\lambda$ & $\Omega(0.3)$ & $\Omega(0.6)$ & $\Omega(0.9)$ \\
\hline 1 & 0.107156478 & 0.103497187 & 0.100099205 \\
\hline 2 & 0.238900952 & 0.228902577 & 0.219832183 \\
\hline 3 & 0.404243315 & 0.383147227 & 0.364602356 \\
\hline 4 & 0.616804097 & 0.575908711 & 0.541468249 \\
\hline 5 & 0.897773718 & 0.820692544 & 0.759471622 \\
\hline 6 & 1.280883932 & 1.136017172 & 1.029732001 \\
\hline 7 & 1.820063752 & 1.546060566 & 1.364936683 \\
\hline 8 & 2.597927127 & 2.079246401 & 1.777777778 \\
\hline 9 & 3.723532349 & 2.762735242 & 2.278240709 \\
\hline 10 & 5.295112884 & 3.61298756 & 2.870624736 \\
\hline
\end{tabular}

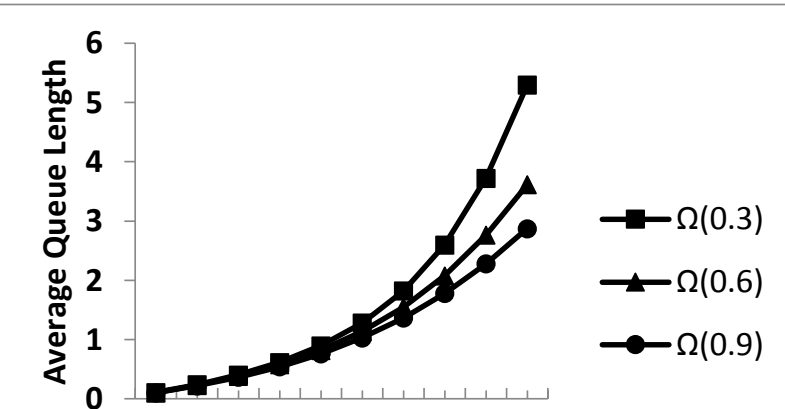


Fig 1

\section{Table :2}

The average length of the system $\mathrm{H}(\mathrm{t})$

is computed for catastrophic effect of $\Omega=0.3,0.6,0.9$ with $\lambda=5$.

\begin{tabular}{|c|c|c|c|}
\hline$\mu$ & $\Omega(0.3)$ & $\Omega(0.6)$ & $\Omega(0.9)$ \\
\hline 6 & 2.455141541 & 1.846464005 & 1.527030955 \\
\hline 7 & 1.766765872 & 1.442734638 & 1.243925591 \\
\hline 8 & 1.349574196 & 1.163331999 & 1.034895452 \\
\hline 9 & 1.081228688 & 0.96539335 & 0.878617872 \\
\hline 10 & 0.897773718 & 0.820692544 & 0.759471622 \\
\hline 11 & 0.765729744 & 0.711548385 & 0.666666667 \\
\hline 12 & 0.666666667 & 0.626871006 & 0.592867953 \\
\hline 13 & 0.589834758 & 0.559551324 & 0.533062874 \\
\hline 14 & 0.528620236 & 0.504900548 & 0.483774357 \\
\hline 15 & 0.478760818 & 0.459734573 & 0.442544832 \\
\hline
\end{tabular}

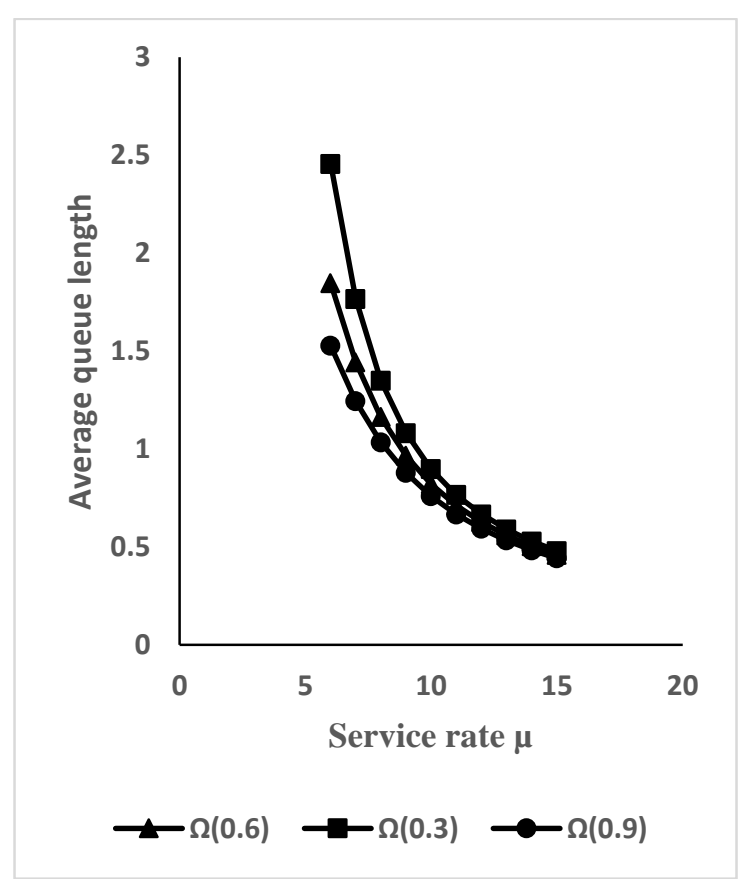

Fig 2 
Table : 3

The stationary probability distribution $\pi_{0}$ for fixed value of $\mu=10$ and $\Omega=0.3, \quad 0.6, \quad 0.9$ for various values of $\lambda$ are computed as follows.

\begin{tabular}{|l|l|l|l|}
\hline$\lambda$ & $\Omega(0.3)$ & $\Omega(0.6)$ & $\Omega(0.9)$ \\
\hline 1 & 0.903214694 & 0.906209831 & 0.909008928 \\
\hline 2 & 0.807167029 & 0.813734155 & 0.819784896 \\
\hline 3 & 0.71212729 & 0.722988834 & 0.732814212 \\
\hline 4 & 0.618504123 & 0.634554523 & 0.648732142 \\
\hline 5 & 0.526933212 & 0.549241553 & 0.568352446 \\
\hline 6 & 0.438426518 & 0.6816103 & 0.49267588 \\
\hline 7 & 0.354601913 & 0.392763634 & 0.422844302 \\
\hline 8 & 0.277937814 & 0.324754784 & 0.36 \\
\hline 9 & 0.21170597 & 0.265764115 & 0.305041664 \\
\hline 10 & 0.158853387 & 0.216779254 & 0.258356226 \\
\hline
\end{tabular}

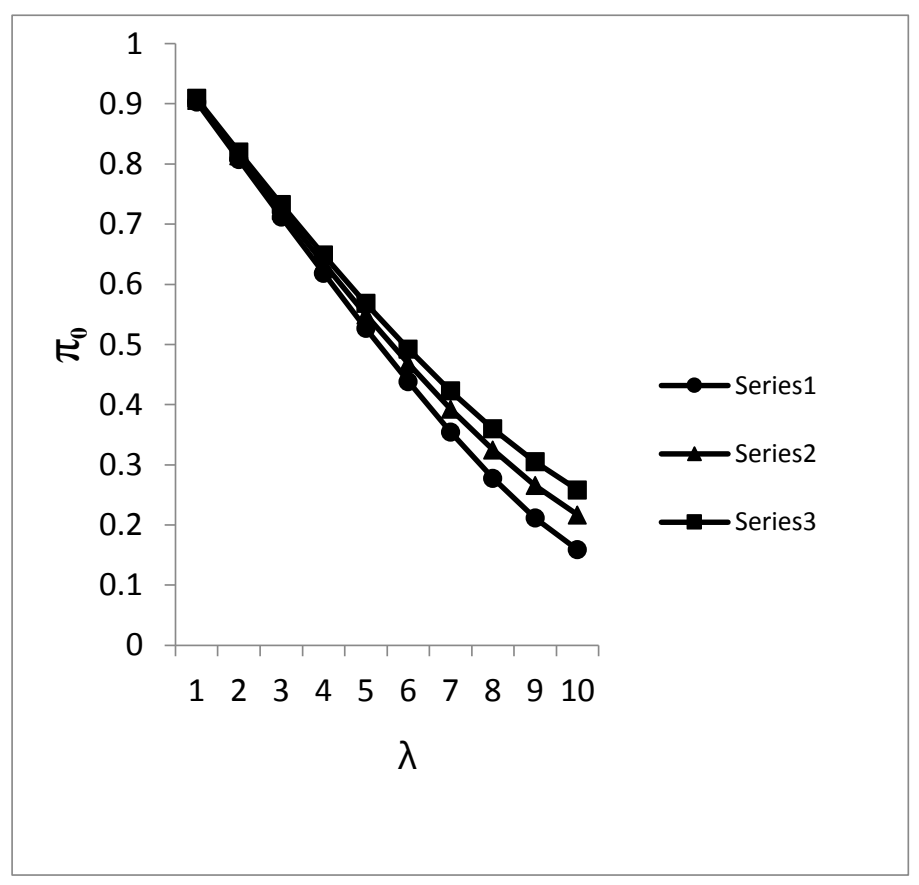

\section{Conclusion:}

Here we derive the probability of n' the system. The numerical

number of customers in the system and no customer in examples shows when the arrival rate 
increases

the average

queue

length increases (as in Fig-1).The increase in service rate decreases the average the queue length (as in Fig-2). As the arrival rate increases the stationary probability of no customers in the system decreases (as in Fig-3). It shows the correctness of the model.

\section{References:}

[1] A.K.Erlang, The Theory of probabilities and Telephone conversations, Nyt Jindsskriff for Mathematic, B 20, 33-39 (1909).

[2] X.Chao, A queueing network model with Operation Research Letters,18,pp.75-79.

catastrophes and product form solution,

[3] Gautam Choudhury and MadhuChanda Paul, A Two phase queueing system With Bernoulli feedback, International Journal of Information and Management Sciences, Vol 16, pp.35-52, 2005.

[4] N.K.Jain and R.kumar " M/G/1 Queue with Catastrophes",Indian Journal of Mathematics and Mathematical Sciences,1(1),pp.45-50,2005.

[5] B.Krishnakumar and S.Pavai Madheswari ,Transient Analysis of an M/M/1 Queue Subject to Catastrophes and Server Failures, Journal of Stochastic Analysis and Applications, Vol 23, pp.329-340, 2005.

[6] A.Santhakumaran, and S.Shanmugasundaram, Preparatory Work on Arrival Customers with a Single Server Feedback Queue.Journalof Information Management Sciences Vol.19, No.2, pp.301-313, June, 2008.

[7] A. Santhakumaran, and V.Thangaraj., A singleserver queue with impatien and feedback customers, International Journal of Information and Management Sciences, Vol.11, pp.71- 79,2000.

[8] L.Takacs,A single Server Queue with feedback, The Bell System Technical Journal, Vol.42,pp. 505-519, 1963.

[9] S.Shanmugasundaram and S.Chitra,Time Dependent Solution of Single Server Feedback Queue Customer has a Service With and Without Preparatory Work when CatastrophesOccur ,I nternational Journal of Applied Engineering Research, ISSN 0973-4562 Vol.11 No.1,2016.

[10] Krishna Kumar,D.Arivudainambi ,Transient Solution of an M/M/1 Queue with catastrophes Computer and Mathematics with Applications, 40(2000) pp:1233-1240.

[11] P.R. Parthasarathy, A transient solution to a M/M/1 queue, a simple Approach, (Adv.Appl.Prob.19(1987) pp: 997-998.

[12] V.Thangraj, S.Vanitha, M/M/1 queue with feedback a continued fraction approach, International Journal of Computational and Applied Mathematics, 5(2010),129-139.

[13] A.Santhakumaran ,K.S.Ramasamy and S.Shanmugasundaram, A single Server queue with instantaneous Bernoulli feedback and setup time,Proceedings of National conference on the emerging trends in Pure and Applied Mathematics, St Xavier College (Autonomous) ,Palayamkottai, pp.58-69, 2005.

[14] V.M. Chandrasekaran, M.C.Saravanarajan, Transient and Reliability analysis of M/M/1 feedback queue subject to catastrophes, server failures and repairs Volume 77, No.5 (2012) pp: 605-622.

[15] B.Krishnakumar, A.Krishnamoorthy, S.Pavai Madheswari and S.Sadiq Basha Transient analysis of a single server queue with catastrophes, failures and repairs, Queueing Syst 56:133-141,2007. 